\title{
Ethanol Extract of the Infructescence of Platycarya strobilacea Sieb. et Zucc. Induces Methuosis of Human Nasopharyngeal Carcinoma Cells
}

\author{
Jinkun Liu, ${ }^{1}$ Min Ying, ${ }^{2}$ Bin Wu $\mathbb{D}^{3},{ }^{3}$ and Chaomei Fu $\mathbb{D}^{1}$ \\ ${ }^{1}$ College of Pharmacy, Chengdu University of Traditional Chinese Medicine, Chengdu 611137, China \\ ${ }^{2}$ Chongqing Key Laboratory of Translational Research for Cancer Metastasis and Individualized Treatment, \\ Chongqing University Cancer Hospital, Chongqing 400030, China \\ ${ }^{3}$ Chongqing Key Laboratory of Traditional Chinese Medicine to Prevent and Treat Autoimmune Diseases, \\ Chongqing Traditional Chinese Medicine Hospital, Chongqing 400021, China \\ Correspondence should be addressed to Bin Wu; wuubinn@126.com and Chaomei Fu; chaomeifu1@163.com
}

Received 20 November 2019; Revised 3 April 2020; Accepted 8 April 2020; Published 29 April 2020

Academic Editor: Maria T. Cruz

Copyright @ 2020 Jinkun Liu et al. This is an open access article distributed under the Creative Commons Attribution License, which permits unrestricted use, distribution, and reproduction in any medium, provided the original work is properly cited.

\begin{abstract}
The infructescence of Platycarya strobilacea Sieb. et Zucc. (PS) has been used in the treatment of rhinitis and sinusitis in clinical practice. Our preliminary study showed that an ethanol extract of the infructescence of PS (EPS) had significant antinasopharyngeal carcinoma (NPC) effects in vitro. However, the mechanism underlying the NPS cell death induced by EPS remains unclear. The aim of the present study was to investigate the inhibitory effects of EPS on NPC cells and to elucidate the underlying mechanism. The effects of EPS on NPC cells were investigated in CNE1 and CNE2 cells in vitro. In EPS-treated cells, the cell morphological changes were evaluated through fluorescence microscope, transmission electron microscopy, and flow cytometry. The underlying mechanism was analyzed via network pharmacology and further verified by western blot analysis. The anticancer effects of EPS were associated with the generation of CNE1 and CNE2 cell fusion and vacuoles, the perturbation of lysosomal vesicle transportation, and the induction of methuosis. The network pharmacology and western blot results indicated that the effect of EPS in NPC cells might be achieved via regulation of the Ras proto-oncogene (RAS)/mitogen-activated protein kinase (MAPK) signaling pathway and the transcription factor c-Fos proto-oncogene (c-FOS) and its downstream genes. EPS induces NPC cell death through methuosis. The mechanism might be related to regulation of the transcription factor c-FOS and its downstream genes.
\end{abstract}

\section{Introduction}

Nasopharyngeal carcinoma (NPC) is a malignant tumor derived from human nasopharyngeal epithelial tissue. One report estimated that 129,079 new cases of NPC and 72,987 NPC-related deaths occurred worldwide in 2018 [1]. The number of NPC patients diagnosed in China within the last 5 years reached 138,500 [2]. At present, the clinical treatment of NPC is mainly based on radiotherapy supplemented by chemotherapy, and no specific drugs for this disease are available [3]. Therefore, identification of new therapeutic targets for drugs, which will help improve the cure and survival rates of NPC and enhance patient quality of life, is important.
Platycarya strobilacea Sieb. et Zucc. (PS) has been widely recognized as a medicinal plant from China with various beneficial effects. The infructescence of PS is believed to eliminate toxic heat, activate blood circulation, relieve swelling, eliminate pus, and ameliorate pain [4-6]; it has also been used in NPC treatment [7]. The Chinese herbal medicine Xiangju capsule, which includes this infructescence as its main component, has been applied in the clinical treatment of rhinitis and sinusitis for more than 20 years. This treatment can induce human leukocytes to produce interferon and improve immunity [8]. The main constituents identified from this infructescence are polyphenols, ellagitannins, and flavone-related compounds [9]. These components include ellagic acid, gallic acid, and ursolic acid, 
which have antioxidative and anti-inflammatory effects $[10,11]$. Our previous experimental study found that ethanol extract of PS (EPS) induced CNE1 and CNE2 cell death, which was similar to methuosis. Methuosis is a form of cell death that ultimately leads to rupture through the production of many intracellular vesicles [12, 13].

However, to our knowledge, the antitumor properties of EPS have not been investigated. We conducted the present research to investigate the inhibitory effect of EPS on NPC cells and to elucidate the intracellular pharmacological mechanism.

\section{Materials and Methods}

2.1. Plant Material. The infructescence of PS was collected in August of 2016 in the vicinity of Dayuanzi Village, Qikou Town, Lueyang County, Hanzhong City, Shanxi Province, China (position: latitude $33.183675^{\circ}$, longitude $106.358065^{\circ}$ ).

Plant material $(4500 \mathrm{~g})$ with the seeds removed was smashed with a 60 mesh sieve. Powder was extracted with $13500 \mathrm{~mL}$ of $95 \%(\mathrm{v} / \mathrm{v})$ ethanol in a shaker bath set at $30^{\circ} \mathrm{C}$ for $0.5 \mathrm{~h}$, and this process was repeated three times. Ethanol was removed from the combined filtrate at $45^{\circ} \mathrm{C}$ using a rotary evaporator. A total of $180 \mathrm{~g}$ of extract was obtained after the aqueous phase, and the yield was $4.5 \%$. A voucher specimen (No. 20160801) was deposited in the Chinese medicine preparation laboratory. HPLC was used to identify the active ingredients in the EPS (Supplemental Table 1).

2.2. Chemicals and Reagents. Methyl thiazolyl tetrazolium (MTT) was purchased from Sigma (Sigma-Aldrich, Inc., St Louis, Missouri, USA). LysoTracker Green DND-26 (L7526) and Hoechst 33342 (R37605) were purchased from Invitrogen (Life Technologies, Shanghai, China). An Annexin V-FITC Apoptosis Kit (556547) and a Cell Cycle Detection Kit (340242) were purchased from BD (BD Biosciences, Inc., San Jose, California, USA). The following antibodies were used in this work: HRas proto-oncogene (HRAS, ab32417), Rac family small GTPase 1 (RAC1, ab155938), coiled-coil domain-containing protein 42 (CDC42, ab187643), Raf-6 proto-oncogene (RAF6, ab131261), member of the RAS oncogene family 7 (RAB7, ab137029), Ras homolog gene family member A (RhoA, ab187027) (Abcam, Cambridge, UK), extracellular regulated protein kinase $1 / 2$ (ERK1/2, 137F5, 4695), caspase-3 and cleaved caspase-3 (D3R6Y, 14220), phosphorylated ERK1/2 (p-ERK1/2, Thr202/Tyr204, 4370), c-Fos proto-oncogene (c-FOS, 9F6, 2250; Cell Signaling Technology, Boston, Massachusetts, USA), and glyceraldehyde-3-phosphate dehydrogenase (GAPDH, 10494-1-AP). Horseradish peroxidase-labeled secondary antibodies (SA00001-2; Proteintech Group, Inc., Chicago, Illinois, USA) were also used. $\beta$-sitosterol (22564) and EHT 1864 (HY-16659, an inhibitor of RAC1) were purchased from MedChemExpress (Monmouth Junction, New Jersey, USA). EHT 1864 was dissolved in dimethyl sulfoxide (DMSO) at $10 \mathrm{mM}$ and stored at $-20^{\circ} \mathrm{C}$. Gallic acid (110831-201605), ellagic acid (111959-201602), and ursolic acid (110742-201421) were obtained from the National Institutes for Food and Drug Control (Beijing, China).

2.3. Cell Culture. The CNE1 and CNE2 cell lines were obtained from the Cell Bank of the Chinese Academy of Science (Shanghai, China). The NP69 cell line was provided by the University of Hong Kong. The cells were maintained in RPMI 1640 medium supplemented with $10 \%$ fetal bovine serum (FBS) (Biological Industries, Inc., Kibbutz Beit Haemek, Israel) at $37^{\circ} \mathrm{C}$ in a humidified chamber containing $5 \% \mathrm{CO}_{2}$.

2.4. Cell Viability Assays. Cell viability was quantified by MTT assays. CNE1 and CNE2 cells were plated at a density of $2.5 \times 10^{3}$ cells/well in 96-well plates with eight replicates for each condition. After $24 \mathrm{~h}$, various concentrations of EPS $(0,2.5,5,10,15,20,30$, and $50 \mathrm{mg} / \mathrm{mL})$ were added to each well, and the plates were incubated for $24 \mathrm{~h}$. Then, $5 \mathrm{mg} / \mathrm{mL}$ of MTT solution was added at $20 \mu \mathrm{L} /$ well and incubated for $4 \mathrm{~h}$ at $37^{\circ} \mathrm{C}$. At the end of the incubation, the MTT-formazan was solubilized in DMSO, and the absorbance was determined with a 96-well plate reader at $490 \mathrm{~nm}$.

2.5. Apoptosis Assay. The cancer cells were seeded into sixwell plates at a density of $2.5 \times 10^{5}$ cells/well and then treated with EPS $(1.0 \mathrm{mg} / \mathrm{mL})$. After $24 \mathrm{~h}$ of incubation, apoptosis was evaluated using an Annexin $\mathrm{V}$-Fluorescein Isothiocyanate (Annexin V-FITC) Kit following the manufacturer's instructions.

2.6. Cell Cycle Analysis. The cancer cells were seeded into $10 \mathrm{~cm}$ plates in $1.0 \mathrm{mg} / \mathrm{mL}$ EPS and RPMI 1640 containing $10 \%$ FBS. After $24 \mathrm{~h}, 5 \times 10^{6}$ cells were harvested, rinsed with cold PBS, and fixed with $75 \%$ ice-cold ethanol for $24 \mathrm{~h}$ at $4^{\circ} \mathrm{C}$. Then, the fixed cells were washed with cold PBS and incubated with propidium iodide (PI, $10 \mu \mathrm{g} / \mathrm{mL}$ ) and RNase A $(0.5 \mathrm{mg} / \mathrm{mL})$ for $30 \mathrm{~min}$ at $37^{\circ} \mathrm{C}$. The procedure was the same for the control group and the experimental group after the cells were recultured in complete culture medium. The DNA content of the labeled cells was quantified by FlowJo 7.6.1 software.

2.7. Transient Transfection with the RAC1 Inhibitor EHT 1864. Twenty-four hours before transfection, the CNE1 and CNE2 NPC cells were plated onto $10 \mathrm{~cm}$ plates at $50 \%$ confluence. EHT 1864 at $40 \mu \mathrm{M}$ was added to the cells for $24 \mathrm{~h}$, and the protein levels were then detected by western blot analysis.

2.8. Live Cell Imaging with Fluorescent Tracers. CNE1 and CNE2 cells were seeded in 6-well plates at a density of $2 \times 10^{5}$ cells/well. Labeling of intracellular acidic compartments with LysoTracker Green DND-26 and staining for EPS activity were performed as described previously. Hoechst $33342(50 \mu \mathrm{g} / \mathrm{mL})$ was used to label the nucleus for $15 \mathrm{~min}$ at $37^{\circ} \mathrm{C}$ with protection from light. Fluorescence intensity was quantified by ImageJ. 
2.9. Transmission Electron Microscopy (TEM). Human CNE1 and CNE2 cells were exposed to $1.0 \mathrm{mg} / \mathrm{mL}$ EPS for $24 \mathrm{~h}$, treated with $2 \%$ citrate fixative, dehydrated with different concentrations of ethanol, infiltrated with acetone, sliced after embedding, double stained with uranyl acetate and lead citrate, and examined with H-7650B TEM (Hitachi, Hitachinaka, Japan).

2.10. Pathway Enrichment Analysis. Gene ontology (GO) functional and pathway enrichment analyses of differentially expressed genes (DEGs) were carried out using the clusterProfiler package of $R$ [14]. The GO terms and pathway terms with adjusted $P$ values $<0.05$ were selected. The search tool for the retrieval of interacting compounds/proteins in the traditional Chinese medicine systems pharmacology database and analysis platform (TCMSP, version 2.3) was used for the prediction of compound-protein interaction information [15]. We set the criteria of oral bioavailability (OB) greater than $40 \%$ and drug-likeness (DL) greater than 0.18 .

2.11. Western Blot Analysis. The cells were lysed in RIPA buffer (70-WB020, MultiSciences, Hangzhou, China). The protein concentration was detected by bicinchoninic acid assays (5000002, Bio-Rad, Hercules, California, USA). The total protein concentration was determined, and the protein samples were separated by SDS-polyacrylamide gel electrophoresis. Nonspecific binding was blocked with 3\% bovine serum albumin in Tris-buffered saline ( $\mathrm{pH} 7.5)$; subsequently, the membranes were incubated with primary antibodies at $4^{\circ} \mathrm{C}$ and then with the corresponding horseradish peroxidase-labeled secondary antibodies. The immunoreactive bands were detected using an enhanced chemiluminescent detection (ECL) kit (70-P1425, MultiSciences, Hangzhou, China).

2.12. Statistical Analyses. The experiments presented are representative of at least three independent repetitions. All data were analyzed using $R$ (3.6.1) and SPSS 20.0 software. The data are presented as the mean \pm SD. A two-tailed Student's $t$-test was used for comparisons of the means of two independent groups. ANOVA was used for comparisons of more than two groups. $P<0.05$ was considered significant.

\section{Results}

3.1. EPS Inhibited NPC Cell Viability. To examine the effect of EPS on cell proliferation, we treated CNE1 and CNE2 cells with multiple concentrations of EPS. As shown in Figure 1(a), the group treated with the lowest concentration of EPS $(0.5 \mathrm{mg} / \mathrm{mL})$ showed a significant difference compared with the control group $(P<0.01)$. The IC50 values of the CNE1 and CNE2 cell lines for EPS were 1.025 and $1.109 \mathrm{mg} / \mathrm{mL}$, respectively. NPC cell viability decreased as the EPS concentration increased. A concentration $(1.0 \mathrm{mg} / \mathrm{mL})$ that showed a moderate antiproliferative effect was used for the subsequent experiments.

Next, the EPS-induced loss of cell viability was confirmed using an Annexin V-FITC/PI-binding assay. Compared with the control cells, EPS-treated cells did not show an increased apoptotic fraction, indicating that EPS suppressed cell proliferation by other cell death mechanisms, Figure 1(b).

3.2. EPS Regulated the Cell Cycle. As shown in Figure 1(c), after treatment with $1.0 \mathrm{mg} / \mathrm{mL}$ EPS for $24 \mathrm{~h}$, the cell population in the $G 0 / G 1$ phase increased to $71.15 \%$ for CNE1 cells and decreased to $43.86 \%$ for CNE2 cells compared with that of the control group. These data indicated that EPS blocked the cell cycle transition from the $G 1$ phase to the $S$ phase in the CNE1 cell line and the $G 2$ phase in the CNE2 cell line.

3.3. EPS-Induced Cell Methuosis. Interestingly, all of the cells treated with EPS $(1.0 \mathrm{mg} / \mathrm{mL})$ for $24 \mathrm{~h}$ showed methuosislike features [16], such as the formation of multiple protrusions in the cell membrane, and the protrusions between the cells were constantly in contact. Many vesicles were produced in the cells, and the multiple vesicles fused into larger vesicles. This change led to extensive cellular vacuolization and ultimately cell death. EHT 1864 is a small molecule inhibitor of RAC1 signaling. This inhibitor did not prevent EPS-induced cell vesicle production. However, this phenomenon was not observed in normal human epithelial NP69 cells given the same treatment (Figure 2(a)).

TEM analysis of cells treated with EPS showed that the cell membranes budded to form bulges, and the bulges formed multiple small vesicles, which then coalesced into a larger structure; these changes resulted in cell membrane rupture (Figure 2(b)). The transparent vesicles in the cells have a single layer membrane, do not contain cytoplasmic components or organelles, and are distinct from the autophagosomes surrounded by a bilayer membrane formed by autophagy. The morphological characteristics of the dying cells were inconsistent with those of various forms of cell death, such as apoptosis, autophagy, and pyroptosis. The TEM results were consistent with the findings of cells undergoing methuosis.

Then, we tested the relationship between lysosomes and methuosis. Lysosomes were visualized by LysoTracker Green DND-26. We found that, in the EPS group, the number and fluorescence intensity of cells were reduced. Intensity quantification revealed that EPS groups have about $29 \%$ and $41 \%$ reduction of LysoTracker Green DND-26 staining, respectively, compared to control groups (Figure 3). LysoTracker Green DND-26's fluorescence decreases in alkaline environments, respectively. The results suggested that the $\mathrm{pH}$ value of cells increased after adding EPS.

3.4. Expression Profiles of NPC Cells after EPS Treatment. To identify the potential genes affected by EPS treatment, we combined $\mathrm{OB}$ screening and DL evaluation to identify the 

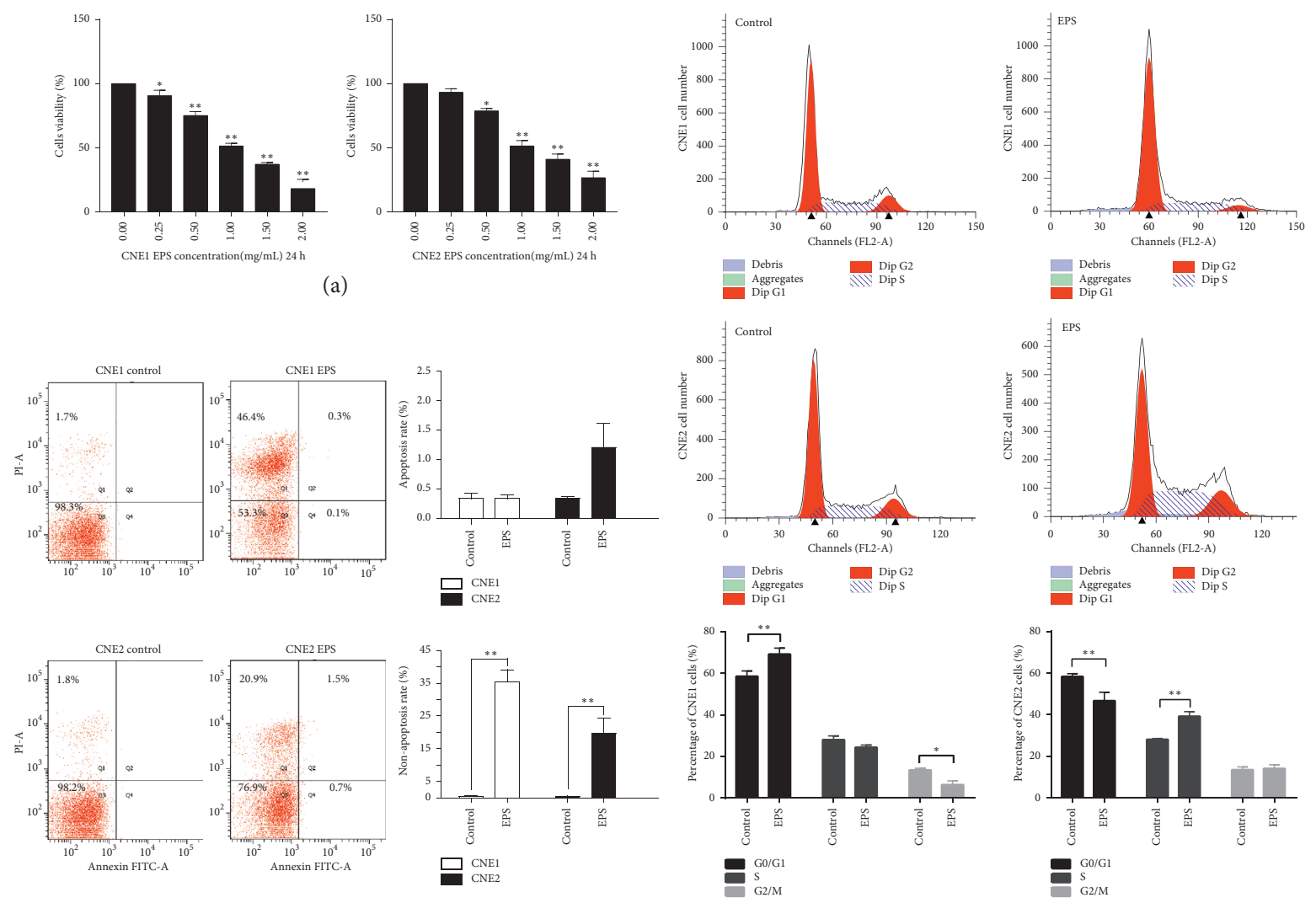

(b)

(c)

FIGURE 1: Effects of EPS on NPC cell viability and the cell cycle. (a) CNE1 and CNE2 cells were treated with various concentrations (0-2.0 mg/mL) of EPS for $24 \mathrm{~h}$. Cell viability was detected using an MTT assay. (b) NPC cells were subjected to $1.0 \mathrm{mg} / \mathrm{mL}$ EPS for $24 \mathrm{~h}$ and then assessed for apoptotic cell death by flow cytometry. The total percentages of apoptotic cell death and nonapoptotic cell death were calculated, as described in the methods. (c) Cell cycle analysis of CNE1 and CNE2 cells treated with the control and $1.0 \mathrm{mg} / \mathrm{mL}$ EPS for $24 \mathrm{~h}$. Representative histograms show the distribution of cells in the cell cycle for each treatment group. Data are expressed as the mean \pm SD of three independent experiments, ${ }^{*} P<0.05,{ }^{* *} P<0.01$ vs the control group, as analyzed by Student's $t$-test.

active compounds in EPS, as shown in Figure 3(a). Five compounds (ursolic acid, ellagic acid, gallic acid, 3,3'dimethoxy ellagic acid, and beta-sitosterol) were widely distributed in EPS. The contents of gallic acid, $\beta$-sitosterol, ellagic acid, and ursolic acid in EPS were $0.72 \%, 1.85 \%$, $4.99 \%$, and $1.88 \%$, respectively (Supplemental Figure 1 ). The total content of the four components in the EPS was 9.44\%. We screened 109 candidate targets by TCMSP, explored the biological networks, and analyzed the relationships between the functional groups (Figure 4(a)). The affected genes that showed GO enrichment were classified into three categories: molecular functions (MF), cellular component (CC), and biological processes (BP) (Figure 4(b)). The genes upregulated by more than twofold had the following functions in sequential order of abundance: G-protein coupled amine receptor activity, G-protein coupled neurotransmitter receptor activity, membrane raft, membrane microdomain, membrane region, plasma membrane raft, transcription factor complex, negative regulation of apoptotic signaling pathway, hepatocellular carcinoma, and pancreatic cancer. Based on the above data, EPS inhibited NPC cells by regulating the major signaling pathways involved in the G-protein coupled receptor channel on the cell membrane and the transcription factor complex. Therefore, we used western blotting to confirm the effects of EPS.

\subsection{The Mechanisms Underlying EPS-Induced Methuosis in} NPC. The mechanisms underlying methuosis are unclear, but previous studies have identified HRAS and RAC1 GTPase as important regulators of this process [16]. To test this hypothesis, we examined the expression levels of HRAS and RAC1 in NPC cells. As shown in Figure 4(c), the levels of the senescence proteins HRAS, RAC1, RAF6, p-ERK1/2, and c-FOS were decreased in the EPS groups. Because caspase- 3 is a critical mediator of apoptosis, the protein levels of caspase- 3 and cleaved caspase- 3 were detected by western blot analysis. There was no elevation of cleaved caspase 3 indicating that EPS-treated NPC cells did not show apoptotic signal.

Furthermore, a systemic search was conducted, and a total of 1491 downstream target genes of c-FOS were defied through the Harmonizome database [17]. The GO BP analysis showed that the genes were enriched in the following pathways: (1) GO: 0060627, regulation of vesiclemediated transport; (2) GO: s0030100, regulation of 


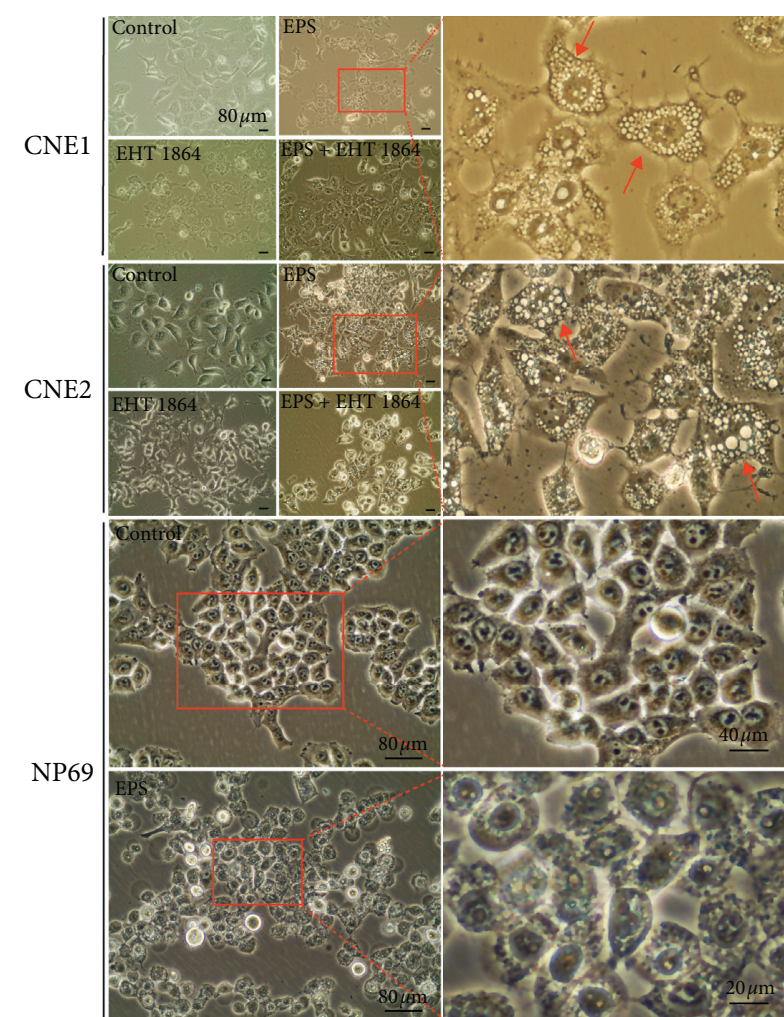

(a)

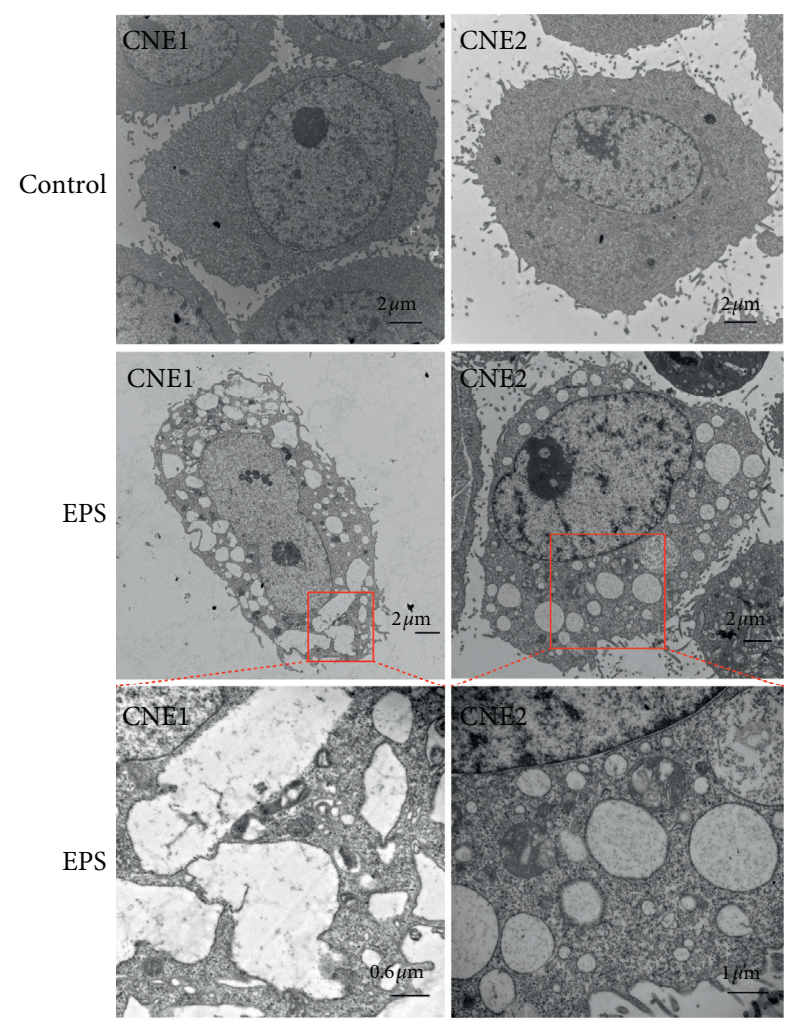

(b)

Figure 2: Cell phenotypic changes after NPC cells and NP69 cells were incubated with $1.0 \mathrm{mg} / \mathrm{mL}$ EPS and $40 \mu \mathrm{M}$ EHT 1840 for $24 \mathrm{~h}$. (a) Microscopic analysis of EPS-treated NPC cells and NP69 cells. (b) CNE1 and CNE2 cells were observed by TEM.

endocytosis; (3) GO: 0909003, vesicle-mediated transport in synapse; (4) GO: 00099560, synaptic membrane adhesion; (5) GO: 0009504, synaptic vesicle cycle; (6) GO: 0022604, regulation of cell morphogenesis; (7) GO: 0048259, regulation of receptor-mediated endocytosis; (8) GO: 0001101, response to acid chemical; and (9) GO: 0098693, regulation of synaptic vesicle cycle (Figures 4(d) and 4(e)). Based on the GO functional analysis, six parts were identified: cell pseudopod formation, cell membrane fusion and vesicle formation, vesicle transport, vesicle growth, stimulation of chemical substances, and membrane regulation Figure 5.

\section{Discussion}

Cells display many different forms of nonapoptotic cell death [18]. Cellular morphological features observed with TEM are commonly used to distinguish between different forms of cell death. Different types of vesicles were found in the cytoplasm in various nonapoptotic forms of cell death, such as autophagy, oncosis, and paraptosis [19]. In this study, we conducted MTT tests to select the appropriate concentration of EPS. The selected concentrations were 0 , $0.25,0.5,1.0,1.5$, and $2.0 \mathrm{mg} / \mathrm{ml}$. The test results showed that $1.0 \mathrm{mg} / \mathrm{ml}$ was the optimal concentration. After addition of $1.0 \mathrm{mg} / \mathrm{ml}$ EPS, we observed the formation of many vesicles, which merged with each other in the cytoplasm to form larger vesicles; furthermore, membrane rupture was observed $24 \mathrm{~h}$ after the EPS treatment. In this report, we identified the signaling pathways underlying a nonapoptotic form of cell death, which can be triggered by constitutive inhibition of the RAS pathway in NPC cell lines. The vacuolization in NPC cells induced by EPS is consistent with previous findings on methuosis [12].

Methuosis is a nonapoptotic form of cell death characterized by cell sprouting that induces a mutual fusion between different cells and the accumulation of vacuoles in the vesicles $[20,21]$. We investigated the possible molecular mechanisms through which EPS causes cell death via methuosis and found that previous studies have shown how RAS is related to methuosis [20-22]. The results indicated that inhibition of HRAS resulted in increased methuosis. HRAS can bind to RAF6-associated vesicles and can be transported by RAF6-mediated localization of vesicles independently of the barrier protein pathway. Inactivation of RAF6 in turn affects membrane trafficking and the actin backbone, resulting in changes in cell morphology, such as vesicle production and cell sprouting [23]. Western blot results also demonstrated that EPS downregulated the protein levels of HRAS and RAF6, which are involved in methuosis of EPS-induced NPC cells.

RAC1 can induce plasma membrane protrusions to form a lamellar layered pseudopod, regulate tumor cell adhesion, and contribute to cell invasion and metastasis [24]. RAC1 also interacts with synaptojanin-2 and decreases clathrinmediated receptor endocytosis, thereby participating in the transport of endocytic vesicles [25]. We pretreated CNE1 


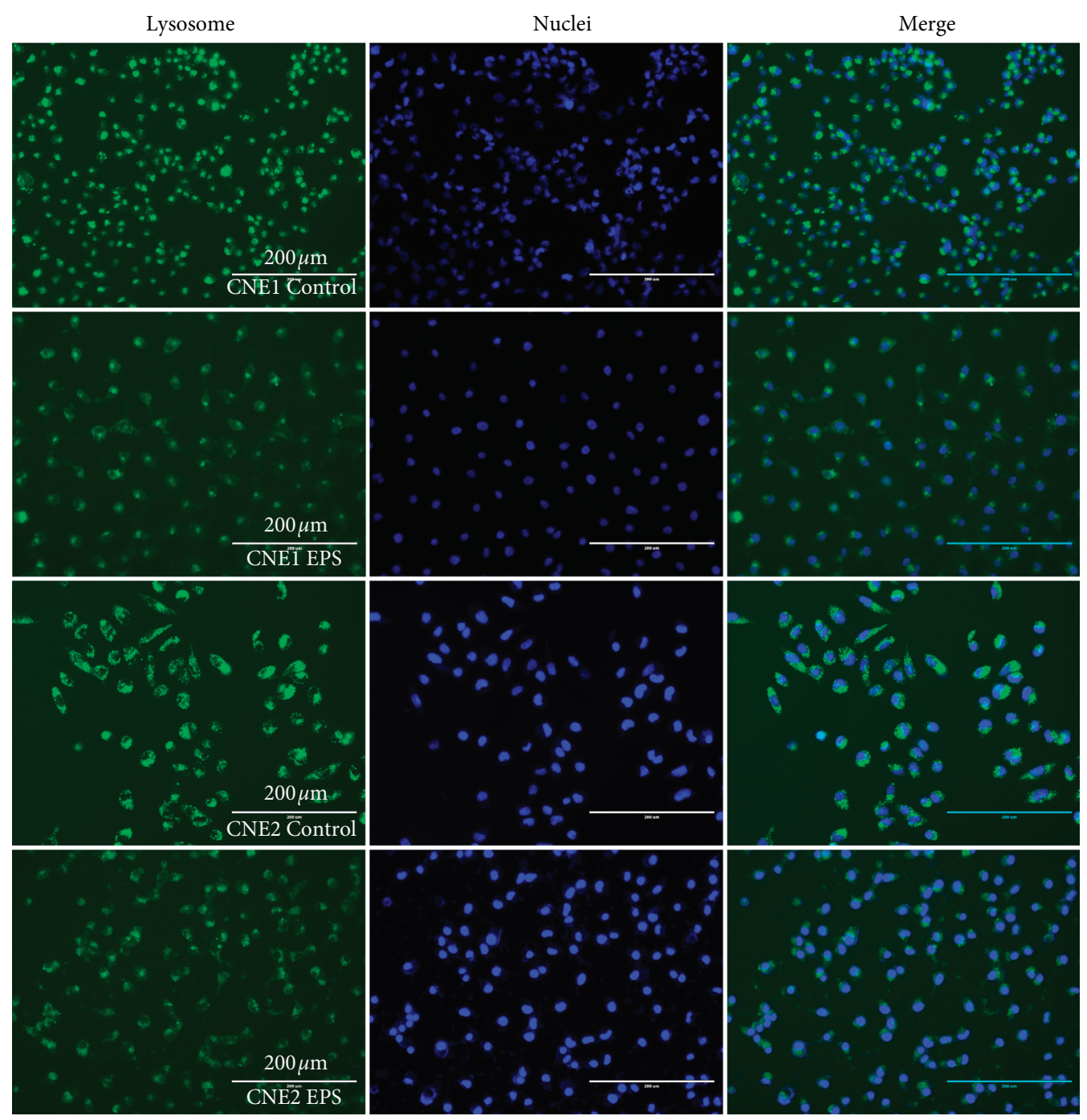

Figure 3: Lysosomes profile in CNE1 and CNE2 after $24 \mathrm{~h}$ exposure to EPS $(1.0 \mathrm{mg} / \mathrm{mL})$. Lysosomes (green) staining was done with LysoTracker Green DND-26, and nuclei (blue) were counterstained with Hoechst $33342(50 \mu \mathrm{g} / \mathrm{mL})$ for $15 \mathrm{~min}$ at $37^{\circ} \mathrm{C}$.

and CNE2 cells with ETH 1864, a specific inhibitor of RAC1 [26]. ETH 1864 could not inhibit the EPS-induced vacuolization of NPC cells. These results indicate that the methuosis induced by EPS in NPC cells does not depend on RAC1.

Studies in the literature have indicated that the Rho small G-protein is involved in important BP, such as cytoskeleton regulation, cell migration, invasion, metastasis, and cell cycle regulation, and thus can promote cell transformation and actin polymerization and remodel the extracellular matrix [27]. The Rho small G-protein family is a member of the RAS superfamily. The best-known RAS homologs, RHOA, RAC1 and $\mathrm{CDC} 42$, have important roles. RHOA regulates cell microfilaments and microtubules, and RAC1 and CDC42 regulate actin activity and cell adhesion, thereby promoting cytoskeletal rearrangement [28]. ERK phosphorylates the CDC42 GTPase protein, and activation of RAC1 and CDC42 promotes the formation of pseudopods at the leading edge [29]. ERK phosphorylates cytoskeletal components in the cytoplasm, such as microtubule-associated protein 1 (MAP-
1), MAP-2, and MAP-4, which are involved in the regulation of cell morphology and the cytoskeletal structure [30]. Activated ERKs phosphorylate many targets, including kinases, transcription factors, and cytoskeletal proteins; these proteins include signal transducer and activator of transcription $1 / 3(\mathrm{STAT1} / 3)$, nuclear factor $-\kappa \mathrm{B}(\mathrm{NF}-\kappa \mathrm{B})$, the oncogene c-MYC, estrogen receptor (ESR), and the transcription factors c-JUN and c-FOS [31]. The downstream target genes of c-FOS were found to be involved in the formation of intracellular vesicles, synaptic membrane adhesion, and cell fusion during cell death.

Simultaneously, in the Cancer Genome Atlas (TCGA) database, a subgroup of head and neck squamous cell carcinoma (HNSC, samples $=508$ ) patients with favorable clinical outcomes showed infrequent copy number alterations that were correlated with activating mutations of HRAS, RAF, RAC1, and ERK1/2 (Supplemental Figure 2). HRAS, RAF, RAC1, and ERK1/2, as targets of tumor-targeting agents, are closely related to the prevention and treatment of tumors [32]. 


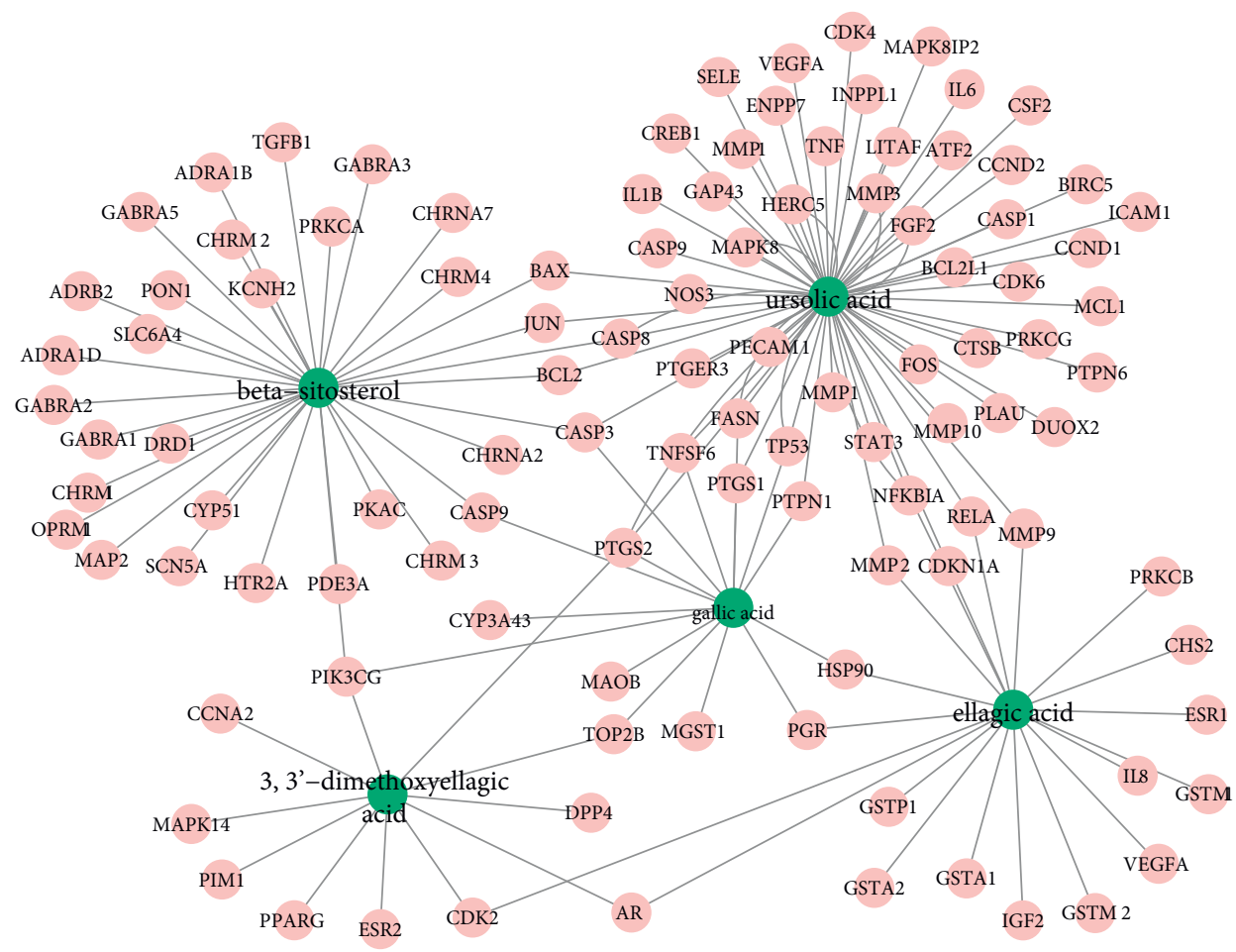

(a)
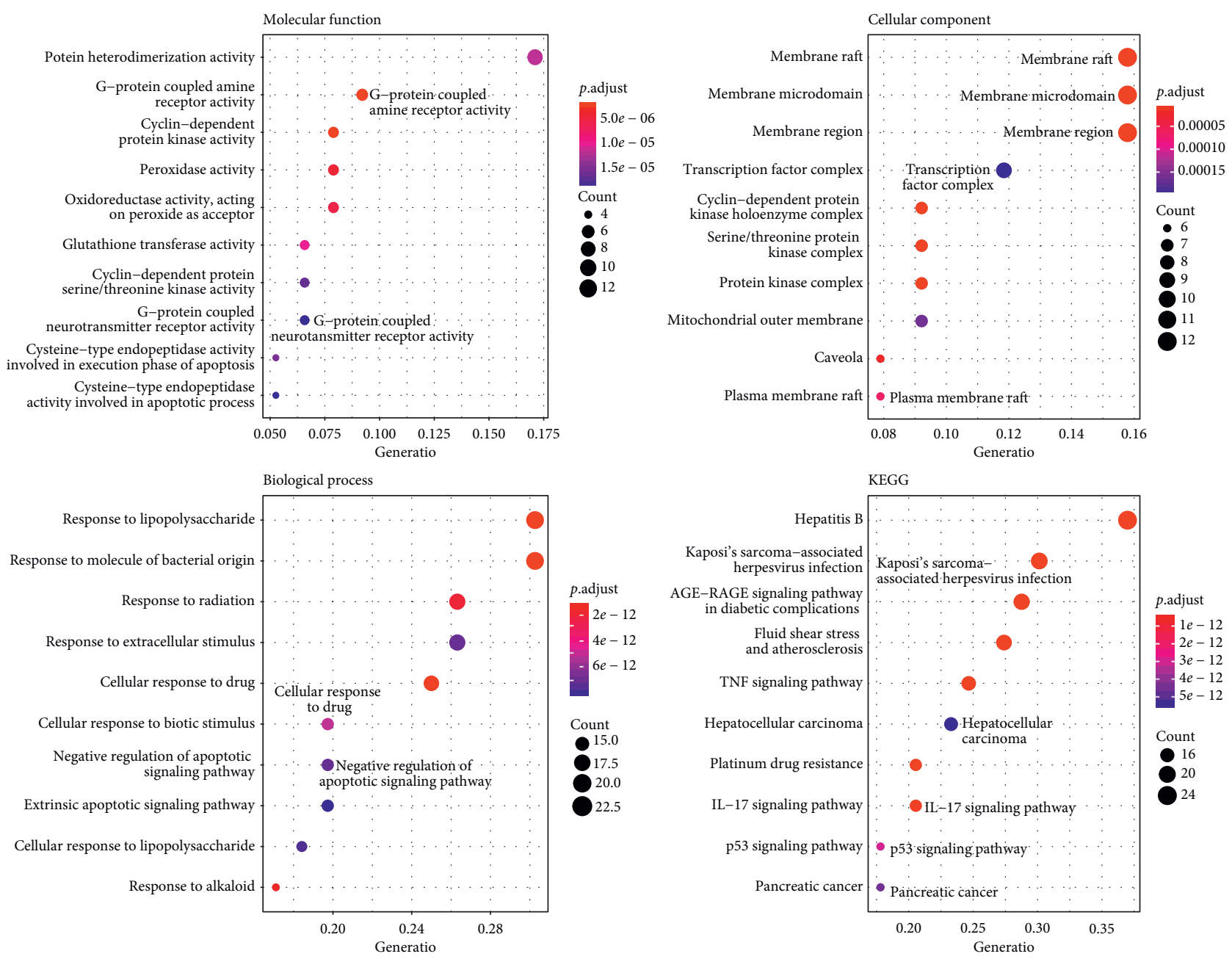

(b)

Figure 4: Continued. 


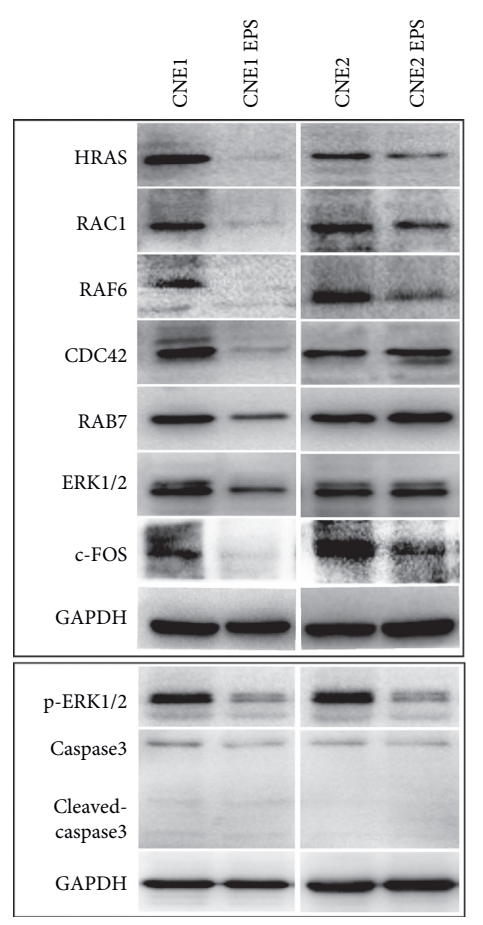

(c)

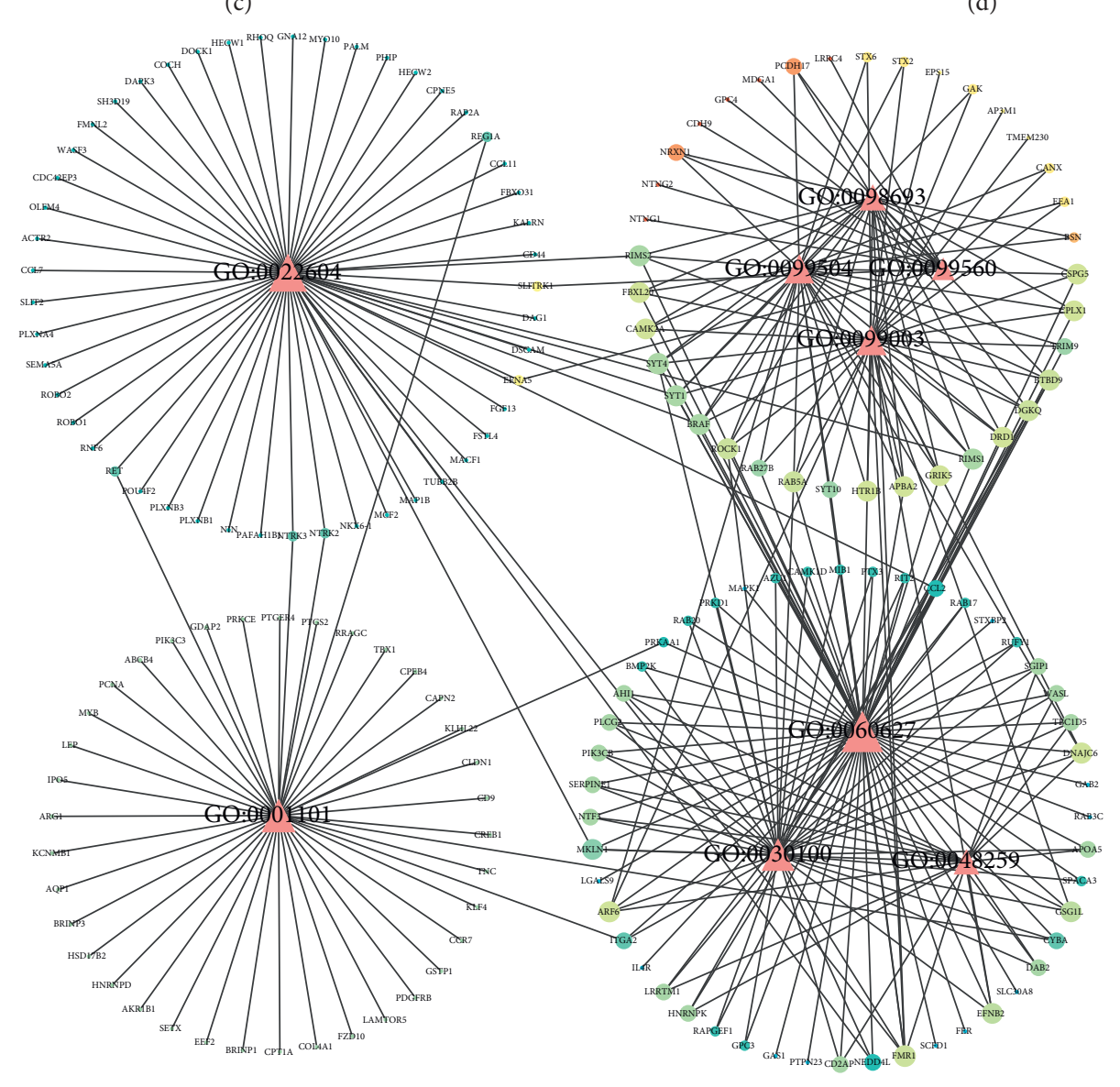

(e)

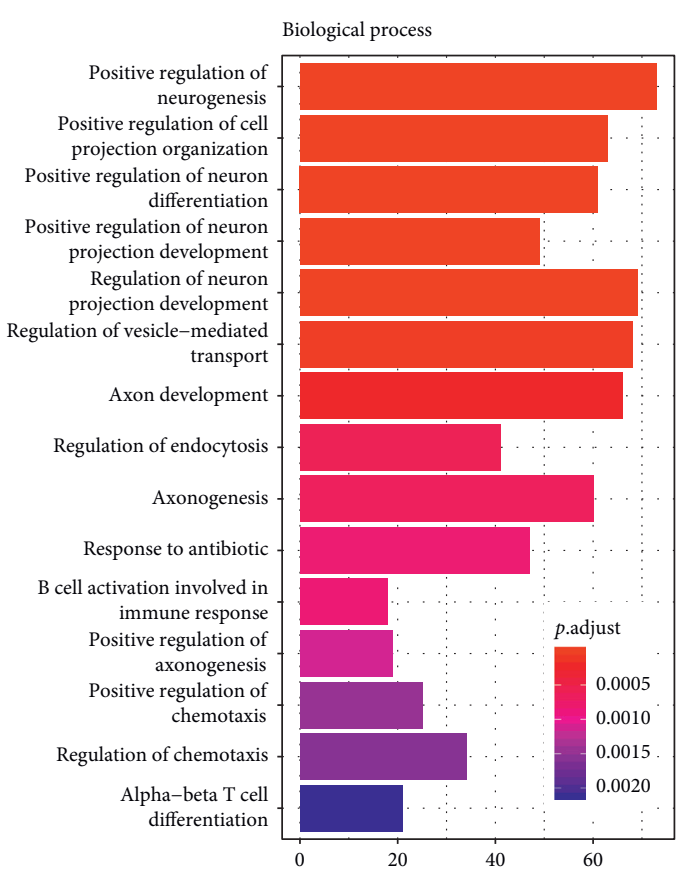

(d)

FIgURE 4: The mechanisms of methuosis induction by EPS in NPC cells, as shown by network pharmacology. (a) Construction of the network map between the active ingredients of EPS and their associated targets. The network was constructed with five candidate compounds and their putative targets, which were constituents of EPS. The green nodes represent candidate compounds, and the targets are indicated by pink circles. (b) GO terms and pathways enriched in the network targets of EPS. The dot size indicates the ratio of genes in the respective pathways to the total number of tested genes, and the dot color illustrates the adjusted $P$ value of the enrichment. (c) Immunoblot analysis of senescence-related proteins in EPS-treated NPC cells. GAPDH was used as a control for these proteins. (d) The GO terms in the biological process categories at all levels enriched in downstream target genes of c-FOS. (e) Construction of the GO BP terms. The histogram was generated by connecting the candidate GO terms and their targeted genes, which were related to methuosis. 


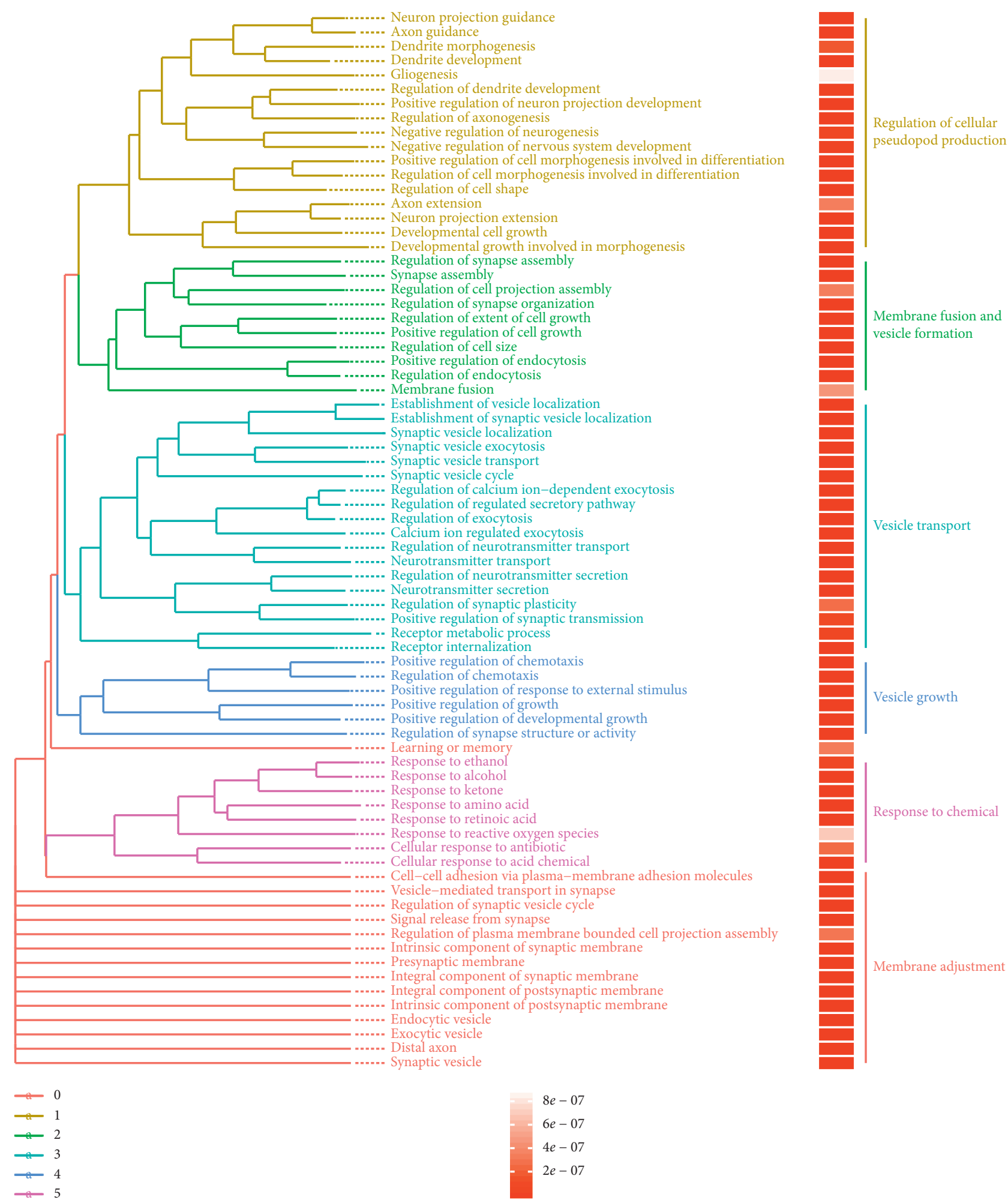

FIGURE 5: GO cluster analysis of the downstream genes of the transcription factor c-FOS during methuosis.

To clarify the role of EPS in NPC cells, we conducted the series of experiments described above. However, our study has some limitations. First, EPS contains many components that could not be completely identified. Second, the downstream target genes require further investigation.
Finally, the drugs that induce methuosis in cells are mainly laboratory-synthesized monomers. Synthesis of these drugs is difficult and, thus, low quantities are available. These molecules are not commercially available, which is the main reason why we could not use these drugs as positive controls. 


\section{Conclusions}

In summary, we identified EPS, a product of natural plants, as a novel allergic inducer that ultimately leads to cell death. EPS can be used to explore the potential relationship between methuosis and anti-NPC effects. Our results also showed that EPS induced the formation of pseudopods, cell fusion, and accumulation of intracellular vesicles in NPC cells, leading to excess vacuoles and cell membrane rupture. Future research will focus on elucidating how EPS regulates the downstream targets of the transcription factor AP-1 and analyses of EPS in vivo. The plants that yield EPS are rich in various resources and inexpensive. We hope that further investigations of this plant and its extract-induced forms of nonapoptotic cell death will be conducted, providing new opportunities for cancer treatment.

\section{Data Availability}

The data used to support the findings of this study are available from the corresponding author upon request.

\section{Conflicts of Interest}

The authors declare no conflicts of interest.

\section{Authors' Contributions}

Jinkun Liu and Min Ying contributed equally to this paper.

\section{Acknowledgments}

This work was supported by the National Natural Science Foundation of China (grant 81904042 and 81673724); the Science and Technology Committee of Chongqing Province (grant cstc2017jcyjAX0369 and cstc2018jxj1130073); and the Chongqing City Health and Family Planning Committee (grant ZY201702038).

\section{Supplementary Materials}

The online version of this article contains Supplementary Materials. Supplemental Figure 1: HPLC fingerprint for EPS. HPLC analysis of EPS was performed with an Agilent XDBODS column $(250 \times 4.6 \mathrm{~mm}, 5 \mu \mathrm{m}$ diameter $)$. EPS was analyzed in an Agilent 1260 series HPLC with a reversed-phase C18 column (Agilent XDB-ODS, $5 \mu \mathrm{m}, 4.6 \times 250 \mathrm{~mm}$ ). (a) Gallic acid standard; (b) EPS sample. (c) ellagic acid; (d) EPS sample; (e) ursolic acid; (f) EPS sample; (g) $\beta$-sitosterol; (h) EPS sample. Supplemental Figure 2: significantly mutated genes in head and neck squamous cell carcinoma (HNSC). The top of the picture shows the total number of mutations per megabase. The left panel indicates the frequency at which each mutation site appears in the sample; the middle panel indicates the mutation information of each gene in each sample; the color indicates the mutation type of the gene specified in the sample. Supplemental Table 1. The content of active ingredients in EPS was determined by HPLC. (Supplementary Materials)

\section{References}

[1] F. Bray, J. Ferlay, I. Soerjomataram, R. L. Siegel, L. A. Torre, and A. Jemal, "Global cancer statistics 2018: GLOBOCAN estimates of incidence and mortality worldwide for 36 cancers in 185 countries," CA: A Cancer Journal for Clinicians, vol. 68, no. 6, pp. 394-424, 2018.

[2] W. Chen, R. Zheng, P. D. Baade et al., "Cancer statistics in China, 2015," CA: A Cancer Journal for Clinicians, vol. 66, no. 2, pp. 115-132, 2016.

[3] X.-R. Tang, Y.-Q. Li, S.-B. Liang et al., "Development and validation of a gene expression-based signature to predict distant metastasis in locoregionally advanced nasopharyngeal carcinoma: a retrospective, multicentre, cohort study," The Lancet Oncology, vol. 19, no. 3, pp. 382-393, 2018.

[4] Y. Liu and Y. Lin, Color Map of the Compendium of Materia Medica, Military Medical Sciences Press, Beijing, China, 2006.

[5] Shanghai Science and Technology Press, Chinese Herbal Medicine, Shanghai Science and Technology Press, Shanghai, China, 2nd edition, 1999.

[6] G. Zhao, D. Shen, and R. Chen, Chinese Medicine Dictionary, Shanghai Scientific Technologic Publisher, Shanghai, China, 2nd edition, 2006.

[7] L. Zhang, Y. Wang, and M. Xu, "In vitro antitumor activities of Platycarya strobilacea Sieb et Zucc infructescence extracts," Tropical Journal of Pharmaceutical Research, vol. 13, no. 6, p. 849, 2014.

[8] Y.-H. Li and L. Peng, "Clinical study on Jin's three-needle plus Xiang Ju capsules for allergic rhinitis," Journal of Acupuncture and Tuina Science, vol. 14, no. 4, pp. 279-283, 2016.

[9] X. Jia, J. Wang, Y. Cheng, X. Zhi, and M. Wang, "Chemical constituents from infructescence of Platycarya strobilacea," Zhong Yao Cai, vol. 39, no. 8, pp. 1786-1788, 2016.

[10] L. Zhang, Y. Wang, and M. Xu, "In vitro and in vivo antioxidant and antimicrobial activities of extracts from Platycarya strobilacea infructescence," Pakistan Journal of Pharmaceutical Sciences, vol. 30, no. 3, pp. 747-753, 2017.

[11] J. H. S. Pang, J. H. Yen, H. T. Wu, and S. T. Huang, "Gallic acid inhibited matrix invasion and AP-1/ETS-1-mediated MMP-1 transcription in human nasopharyngeal carcinoma cells," International Journal of Molecular Sciences, vol. 18, no. 7, p. 1354, 2017.

[12] W. A. Maltese and J. H. Overmeyer, "Methuosis," The American Journal of Pathology, vol. 184, no. 6, pp. 1630-1642, 2014.

[13] Z. Li, N. E. Mbah, J. H. Overmeyer et al., “The JNK signaling pathway plays a key role in methuosis (non-apoptotic cell death) induced by MOMIPP in glioblastoma," BMC Cancer, vol. 19, no. 1, p. 77, 2019.

[14] G. Yu, L.-G. Wang, Y. Han, and Q.-Y. He, "clusterProfiler: an $\mathrm{R}$ package for comparing biological themes among gene clusters," OMICS: A Journal of Integrative Biology, vol. 16, no. 5, pp. 284-287, 2012.

[15] J. Ru, P. Li, J. Wang et al., "TCMSPT: a database of systems pharmacology for drug discovery from herbal medicines," Journal of Cheminformatics, vol. 6, no. 1, p. 13, 2014.

[16] W. A. Maltese and J. H. Overmeyer, "Non-apoptotic cell death associated with perturbations of macropinocytosis," Frontiers in Physiology, vol. 6, no. 38, 2015.

[17] A. D. Rouillard, G. W. Gundersen, N. F. Fernandez et al., "The harmonizome: a collection of processed datasets gathered to serve and mine knowledge about genes and proteins," $D a$ tabase, vol. 2016, Article ID baw100, 2016. 
[18] M. Diederich and C. Cerella, "Non-canonical programmed cell death mechanisms triggered by natural compounds," Seminars in Cancer Biology, vol. 40-41, pp. 4-34, 2016.

[19] S. W. G. Tait, G. Ichim, and D. R. Green, "Die another waynon-apoptotic mechanisms of cell death," Journal of Cell Science, vol. 127, no. 10, pp. 2135-2144, 2014.

[20] N. E. Mbah, J. H. Overmeyer, and W. A. Maltese, "Disruption of endolysosomal trafficking pathways in glioma cells by methuosis-inducing indole-based chalcones," Cell Biology and Toxicology, vol. 33, no. 3, pp. 263-282, 2017.

[21] J. H. Overmeyer, A. M. Young, H. Bhanot, and W. A. Maltese, "A chalcone-related small molecule that induces methuosis, a novel form of non-apoptotic cell death, in glioblastoma cells," Molecular Cancer, vol. 10, no. 1, p. 69, 2011.

[22] C. Commisso, S. M. Davidson, R. G. Soydaner-Azeloglu et al., "Macropinocytosis of protein is an amino acid supply route in Ras-transformed cells," Nature, vol. 497, no. 7451, pp. 633637, 2013.

[23] J. McKay, X. Wang, J. Ding, J. E. Buss, and L. Ambrosio, "HRas resides on clathrin-independent ARF6 vesicles that harbor little RAF-1, but not on clathrin-dependent endosomes," Biochimica et Biophysica Acta (BBA)-Molecular Cell Research, vol. 1813, no. 2, pp. 298-307, 2011.

[24] M. Parri and P. Chiarugi, "Rac and Rho GTPases in cancer cell motilitycontrol," Cell Communication and Signaling, vol. 8, no. 1, pp. 23-36, 2010.

[25] N. Akhtar, W. Li, A. Mironov, and C. H. Streuli, "Rac1 controls both the secretory function of the mammary gland and its remodeling for successive gestations," Developmental Cell, vol. 38, no. 5, pp. 522-535, 2016.

[26] A. Shutes, C. Onesto, V. Picard, B. Leblond, F. Schweighoffer, and C. J. Der, "Specificity and mechanism of action of EHT 1864, a novel small molecule inhibitor of Rac family small GTPases," Journal of Biological Chemistry, vol. 282, no. 49, pp. 35666-35678, 2007.

[27] Y. Egami, T. Taguchi, M. Maekawa, H. Arai, and N. Araki, "Small GTPases and phosphoinositides in the regulatory mechanisms of macropinosome formation and maturation: GTPases and phosphoinositides in macropinocytosis," Frontiers in Physiology, vol. 5, 2014.

[28] A. J. Ridley, "Rho GTPase signalling in cell migration," Current Opinion in Cell Biology, vol. 36, pp. 103-112, 2015.

[29] S. Tanimura and K. Takeda, "ERK signalling as a regulator of cell motility," The Journal of Biochemistry, vol. 162, no. 3, pp. 145-154, 2017.

[30] L. O. Murphy, S. Smith, R.-H. Chen, D. C. Fingar, and J. Blenis, "Molecular interpretation of ERK signal duration by immediate early gene products," Nature Cell Biology, vol. 4, no. 8, pp. 556-564, 2002.

[31] A. S. Dhillon, S. Hagan, O. Rath, and W. Kolch, "MAP kinase signalling pathways in cancer," Oncogene, vol. 26, no. 22, pp. 3279-3290, 2007.

[32] A. A. Samatar and P. I. Poulikakos, "Targeting RAS-ERK signalling in cancer: promises and challenges," Nature Reviews Drug Discovery, vol. 13, no. 12, pp. 928-942, 2014. 